\title{
ADMISSIBLE TRANSLATES OF MEASURES ON A TOPOLOGICAL GROUP
}

By

\author{
Yoshiaki OKAZAKI \\ (Kyushu University)
}

(Received Jan. 18, 1979)

\begin{abstract}
Contents

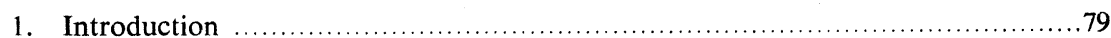

2. Algebraical properties of admissible (singular) translates .........................81

3. Measure theoretical properties of admissible (singular) translates ..................82

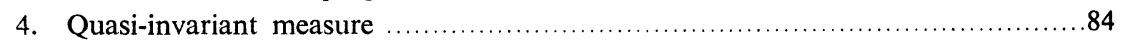

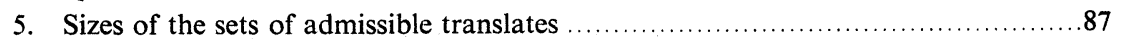

\begin{abstract}
The algebraical and the measure theoretical properties of admissible (singular) translates on a topological group are studied. It is shown the sets of all admissible (singular) translates such as $R A(\mu), L A(\mu), T A(\mu), R E(\mu), L E(\mu), T E(\mu), R S(\mu), L S(\mu)$, $T S(\mu)$, are Borel subsets of the topological group. If $\mu_{1}$ is right quasi-invariant and $\mu_{2}$ is left quasi-invariant then $\mu_{1}, \mu_{2}$ are equivalent. In a locally compact case each right (or left) quasi-invariant measure is equivalent to the Haar measure. For a right quasiinvariant measure $\mu$ on a separable group, a right translation invariant measure $\mu_{0}$ which is equivalent to $\mu$ is constructed. Using this fact the following result is proved, which gives some informations about the measure theoretical size of the set of admissible translates.
\end{abstract}

THEOREM 1. For each Radon probability measure $\mu$ on a separable group $G$, $\mu(R E(\mu))=0$, or $R E(\mu)$ is a locally compact group and the restriction $\mu \mid R E(\mu)$ is equivalent to the Haar measure on $R E(\mu)$.

\section{Introduction}

Let $G$ be a topological group, $\mathscr{B}(G)$ be the Borel field and $M(G)$ be the set of all probability measures on $\mathscr{B}(G)$. For an $a \in G$ let $R_{a}$ (resp. $L_{a}$ ) be the right (resp. left) translation, that is, 


$$
\left.R_{a}(x)=x a \quad \text { (resp. } L a(x)=a x\right) \quad \text { for } \quad x \in G .
$$

For $\mu, v \in M(G)$, we write $\mu \ll v$ (resp. $\mu \perp v$ ) when $\mu$ is absolutely continuous (resp. singular) with respect to $v$. If $\mu$ and $v$ are mutually absolutely continuous (=equivalent) we denote $\mu \sim v$.

Notations. $R A(\mu)=\left\{a \in G ; R_{a}(\mu) \ll \mu\right\}, \quad L A(\mu)=\left\{a \in G ; L_{a}(\mu) \ll \mu\right\}$, $T A(\mu)=R A(\mu) \cap L A(\mu), R E(\mu)=\left\{a \in G ; R_{a}(\mu) \sim \mu\right\}, L E(\mu)=\left\{a \in G ; L_{a}(\mu) \sim \mu\right\}$, $T E(\mu)=R E(\mu) \cap L E(\mu), R S(\mu)=\left\{a \in G ; R_{a}(\mu) \perp \mu\right\}, L S(\mu)=\left\{a \in G ; L_{a}(\mu) \perp \mu\right\}$, $T S(\mu)=R S(\mu) \cap L S(\mu),\left(R_{a}(\mu), L_{a}(\mu)\right.$ are the image measures of $\mu$ by $\left.R_{a}, L_{a}\right)$.

The elements of $R A(\mu)$ (resp. $L A(\mu), T A(\mu)$ ) is called the right (resp. left, two sided) admissible translates of $\mu$. The elements of $R S(\mu)$ (resp. $L S(\mu), T S(\mu)$ ) is called the right (resp. left, two sided) singular translates of $\mu$.

In this paper we study the algebraical and the measure theoretical properties of admissible (singular) translates.

In section 2, we show the sets $R E(\mu), L E(\mu)$ are subgroups. The semigroup properties are also proved such as $\operatorname{TS}(\mu) \cdot \operatorname{TE}(\mu) \subset \operatorname{TS}(\mu), \operatorname{TE}(\mu) \cdot \operatorname{TS}(\mu) \subset \operatorname{TS}(\mu)$.

In section 3 , we investigate the image of admissible (singular) translates by a Borel homomorphism $\psi: G \rightarrow H$. We show, for example, $\psi(R A(\mu)) \subset R A(\psi(\mu))$, $\psi(R E(\mu)) \subset R E(\psi(\mu))$, and $\psi^{-1}(R S(\psi(\mu))) \subset R S(\mu)$. Applying this result we prove if $G$ is abelian and $\mu=v_{1} * v_{2} * \cdots * v_{n}$ then it hold $T A\left(v_{1}\right) \cdot T A\left(v_{2}\right) \cdots \cdot T A\left(v_{n}\right) \subset T A(\mu)$ and $T E\left(v_{1}\right) \cdot T E\left(v_{2}\right) \cdots \cdot T E\left(v_{n}\right) \subset T E(\mu)$.

In the first half of section 4 , we prove the subsets $R A(\mu), R E(\mu), R S(\mu)$ are Borel subsets when $G$ is separable (=second countable). The work of Dubins and Freedman [4] is essential. In the latter half we investigate the quasi-invariant measures. We show if $\mu_{1}$ is right quasi-invariant and $\mu_{2}$ is left quasi-invariant then $\mu_{1}$ is equivalent to $\mu_{2}$. Similar result was given by Umemura [8] in case $G$ is a vector space. If $G$ is a locally compact group then each right quasi-invariant measure is equivalent to the Haar measure.

In section 5 we show the "size" of the set of admissible translates is small in the following sense. For a separable group $G$ and a Radon measure $\mu \in M(G)$ we have $(R E(\mu))=0$, or $R E(\mu)$ is a locally compact group and the restriction $\mu \mid R E(\mu)$ is equivalent to the Haar measure on $R E(\mu)$. The equivalence with the Haar measure was investigated by Brockett [3]. Similar result for $T A(\mu)$ is also given in case $G$ is abelian and $\mu$ is symmetric. If $G$ is a separable vector space and $R E(\mu)$ is a linear subspace, then $R E(\mu)$ is finite dimensional or $\mu(R E(\mu))=0$. This was proved by Umemura [8], Zinn [10]. 


\section{Algebraical properties of admissible (singular) translates}

Proposition 1. (1) The sets $R A(\mu), L A(\mu)$ and $T A(\mu)$ are semigroups.

(2) The sets $R E(\mu), L E(\mu)$ and $T E(\mu)$ are groups.

Proof. The first assertion is easy. To see $R E(\mu)$ is a group, we show $x^{-1} \in R E(\mu)$ for every $x \in R E(\mu)$. For every $A \in \mathscr{B}(G), \mu(A)=0$ is equivalent to $\mu\left(A x^{-1}\right)=0$, hence $\mu(A x)=0$ is equivalent to $\mu(A)=0$ since $\mathscr{B}(G)$ is translation invariant (that is, $\mathscr{B}(G)=\{A x ; A \in \mathscr{B}(G)\}$ ). This shows $R_{x^{-1}}(\mu) \sim \mu$.

Proposition 2. Suppose $a \in R A(\mu)$ and $a^{n}=e$ for some $n$. Then it hold $a \in R E(\mu)$ and each two measures in $\left\{\mu, R_{a}(\mu), R_{a^{2}}(\mu), \ldots, R_{a^{n-1}}(\mu)\right\}$ are equivalent.

Proof. Let $A$ be $R_{a}(\mu)(A)=\mu\left(A a^{-1}\right)=0$. Then we have $\mu\left(A a^{-2}\right)=$ $\mu\left(A a^{-3}\right)=\cdots=\mu\left(A a^{-n}\right)=\mu(A)=0$.

Proposition 3.

(1) $R A(\mu) \cdot R S(\mu) \subset R S(\mu)$

(2) $L S(\mu) \cdot L A(\mu) \subset L S(\mu)$

Proof. (1) Let $a \in R A(\mu)$ and $b \in R S(\mu)$. Since $R_{b}(\mu) \perp \mu$, there is an $A \in \mathscr{B}(G)$ such that $\mu(A)=0, \mu\left(A^{c} b^{-1}\right)=0$. By $R_{a}(\mu) \ll \mu$, we have $\mu\left(A^{c} b^{-1} a^{-1}\right)$ $=R_{a b}(\mu)\left(A^{c}\right)=0$. Thus it holds $a b \in R S(\mu)$.

(2) Let $a \in L S(\mu)$ and $b \in L A(\mu)$. Take an $A \in \mathscr{B}(G)$ so that $\mu(A)=0$, $\mu\left(a^{-1} A^{c}\right)=0$. Since $b \in L A(\mu)$, we have $\mu\left(b^{-1} a^{-1} A^{c}\right)=L_{a b}(\mu)\left(A^{c}\right)=0$, that is, $a b \in L S(\mu)$.

\section{PROPOSITION 4.}

(1) $R S(\mu) \cdot R E(\mu) \subset R S(\mu)$

(2) $L E(\mu) \cdot L S(\mu) \subset L S(\mu)$

Proof. (1) Let $a \in R S(\mu)$ and $b \in R E(\mu)$. By $R_{a}(\mu) \perp \mu$, there is an $A \in \mathscr{B}(G)$ such that $\mu(A)=0$ and $\mu\left(A^{c} a^{-1}\right)=0$. Since $R_{b}(\mu) \sim \mu$ and $0=\mu\left(A b b^{-1}\right)$ $=R_{b}(\mu)(A b)$, we have $\mu(A b)=0$. On the other hand we have $R_{a b}(\mu)\left(A^{c} b\right)$ $=\mu\left(A^{c} b b^{-1} a^{-1}\right)=\mu\left(A^{c} a^{-1}\right)=0$. This shows $R_{a b}(\mu) \perp \mu$, that is, $a b \in R S(\mu)$.

(2) Let $a \in R E(\mu)$ and $b \in L S(\mu)$. Take $A \in \mathscr{B}(G)$ so that $\mu(A)=0, \mu\left(b^{-1} A^{c}\right)$ $=L_{b}(\mu)\left(A^{c}\right)=0$. Since $\mu \sim L_{a}(\mu)$ and $L_{a}(\mu)(a A)=\mu(A)=0$, we have $\mu(a A)=0$. On the other hand we have $L_{a b}(\mu)\left(a A^{c}\right)=\mu\left(b^{-1} a^{-1} a A^{c}\right)=\mu\left(b^{-1} A^{c}\right)=0$. This 
shows $L_{a b}(\mu) \perp \mu$.

Proposition 5.

(1) $T S(\mu) \cdot T E(\mu) \subset T S(\mu)$

(2) $T E(\mu) \cdot T S(\mu) \subset T S(\mu)$

Proof. The assertions follow from propositions 3 and 4 .

\section{Measure theoretical properties of admissible (singular) translates}

Proposition 6. Let $\mu \in M(G)$ and $H$ be a Borel subgroup of $G$ such that $\mu(H)=1$. Then we have $R A(\mu) \cup L A(\mu) \subset H$.

Proof. If there is an $a \in R A(\mu)$ such that $a \notin H$, then we have $\mu\left(H^{c} a^{-1}\right)$ $=R_{a}(\mu)\left(H^{c}\right)=0$ and $H \cap\left(H a^{-1}\right)=\phi$. Thus $\mu(G) \geqq \mu(H)+\mu\left(H a^{-1}\right)=2$, which contradicts to $\mu(G)=1$.

Lemma 1. Let $G, H$ be two topological groups and $\psi: G \rightarrow H$ be a Borel holomorphism. Then we have

(1) $\psi\left(R_{a}(\mu)\right)=R_{\psi(a)}(\psi(\mu))$, and

(2) $\psi\left(L_{a}(\mu)\right)=L_{\psi(a)}(\psi(\mu))$.

Proof. (1) First we remark $\psi^{-1}(A) \cdot a^{-1}=\psi^{-1}\left(A \cdot \psi(a)^{-1}\right)$. In fact if $x \in \psi^{-1}\left(A \cdot \psi(a)^{-1}\right)$ then it holds $\psi(x) \in A \cdot \psi(a)^{-1}$, that is, $\psi(x a)=\psi(x) \psi(a) \in A$. Thus $x a \in \psi^{-1}(A)$ holds, so we have $x \in \psi^{-1}(A) \cdot a^{-1}$. For every $A \in \mathscr{B}(H)$, we have $\psi\left(R_{a}(\mu)\right)(A)=R_{a}(\mu)\left(\psi^{-1}(A)\right)=\mu\left(\psi^{-1}(A) \cdot a^{-1}\right)=\mu\left(\psi^{-1}\left(A \cdot \psi(a)^{-1}\right)\right)=\psi(\mu)$. $\left(A \cdot \psi(a)^{-1}\right)=R_{\psi(a)}(\psi(\mu))(A)$, which shows (1).

(2) is analogous to (1).

Lemma 2. Let $f: G \rightarrow H$ be a Borel mapping and $\mu, v \in M(G)$.

(1) If $\mu \ll v$ then $f(\mu) \ll f(v)$ holds.

(2) If $\mu \sim v$ then $f(\mu) \sim f(v)$ holds.

Proof. (1) Let $A \in \mathscr{B}(H)$ be $f(v)(A)=0$, that is $v\left(f^{-1}(A)\right)=0$. Then it holds $\mu\left(f^{-1}(A)\right)=f(\mu)(A)=0$. This proves (1).

(2) follows from (1).

We consider the image of admissible translates by a Borel homomorphism.

Proposition 7. Let $\psi: G \rightarrow H$ be a Borel homomorphism and $\mu \in M(G)$. Then we have 
(1) $\psi(R A(\mu)) \subset R A(\psi(\mu)), \psi(L A(\mu)) \subset L A(\psi(\mu))$ and $\psi(T A(\mu)) \subset T A(\psi(\mu))$.

(2) $\psi(R E(\mu)) \subset R E(\psi(\mu)), \psi(L E(\mu)) \subset L E(\psi(\mu))$ and $\psi(T E(\mu)) \subset T E(\psi(\mu))$.

If $\psi$ is a Borel isomorphism, then equalities hold.

Proof. The assertion follow from lemmas 1 and 2.

For the singular translates we have the following result (see also Zinn [10]).

Proposition 8. Let $\psi: G \rightarrow H$ be a Borel homomorphism and $\mu \in M(G)$. Then we have $\psi^{-1}(R S(\psi(\mu))) \subset R S(\mu), \psi^{-1}(L S(\psi(\mu))) \subset L S(\mu)$ and $\psi^{-1}(T S(\psi(\mu)))$ $\subset T S(\mu)$. If $\psi$ is a Borel isomorphism, the equalities hold.

Proof. For example we prove $\psi^{-1}(R S(\psi(\mu))) \subset R S(\mu)$. Let $x \in \psi^{-1}(R S$. $(\psi(\mu)))$, that is, $\psi(x) \in R S(\psi(\mu))$. By lemma 1 we have $\psi\left(R_{x}(\mu)\right) \perp \psi(\mu)$. Take a $D \in \mathscr{B}(H)$ such that $\psi(\mu)(D)=0$ and $\psi\left(R_{x}(\mu)\right)\left(D^{c}\right)=0$. Then for $A=\psi^{-1}(D)$ we have $\mu(A)=0$ and $R_{x}(\mu)\left(A^{c}\right)=0$. Thus it hold $R_{x}(\mu) \perp \mu$, hence we have $x \in R S(\mu)$.

Corollary 1. We have the following inclusions; $R A(\mu) \subset \psi^{-1}\left(R S(\psi(\mu))^{c}\right)$, $L A(\mu) \subset \psi^{-1}\left(L S(\psi(\mu))^{c}\right)$ and $T A(\mu) \subset \psi^{-1}\left(T S(\psi(\mu))^{c}\right)$.

Now we consider the convolution of measures when $G$ is abelian. For $\mu, v \in M(G)$, we denote by $\mu * v$ the convolution of $\mu$ and $v$.

Proposition 9. Let $G$ be abelian and $\mu=v_{1} * v_{2} * \cdots * v_{n}, \mu, v_{1}, v_{2}, \ldots, v_{n}$ $\in M(G)$. Then we have

(1) $T A\left(v_{1}\right) \cdot T A\left(v_{2}\right) \cdots \cdot T A\left(v_{n}\right) \subset T A(\mu)$, and

(2) $T E\left(v_{1}\right) \cdot T E\left(v_{2}\right) \cdots \cdot T E\left(v_{n}\right) \subset T E(\mu)$.

Proof. We remark $R A(\mu)=L A(\mu)=T A(\mu)$ and $R E(\mu)=L E(\mu)=T E(\mu)$ since $G$ is abelian. Let $\psi$ be the continuous homomorphism

$$
\psi: G \times G \times \cdots \times G \longrightarrow G, \quad \psi\left(x_{1}, x_{2}, \ldots, x_{n}\right)=x_{1} \cdot x_{2} \cdots \cdot x_{n} .
$$

Now let $v_{1} \otimes v_{2} \otimes \cdots \otimes v_{n}$ be the product measure on $G \times G \times \cdots \times G$, then the image $\psi\left(v_{1} \otimes v_{2} \otimes \cdots \otimes v_{n}\right)$ is the convolution $v_{1} * v_{2} * \cdots * v_{n}=\mu$. For every $a_{i} \in T A\left(v_{i}\right)$, the $n$-tuple $\left(a_{1}, a_{2}, \ldots, a_{n}\right)$ belongs to $T A\left(v_{1} \otimes v_{2} \otimes \cdots \otimes v_{n}\right)$ on $G \times G \times \cdots \times G$. By proposition 7 we have $a_{1} \cdot a_{2} \cdots \cdot a_{n}=\psi\left(\left(a_{1}, a_{2}, \ldots, a_{n}\right)\right) \in T A(\mu)$.

Let $G$ be abelian. We say $\nu \in M(G)$ is a factor of $\mu \in M(G)$ if there exists a $\lambda \in M(G)$ such that $\nu * \lambda=\mu$. 
Corollary 2. Let $G$ be abelian and $v$ be a factor of $\mu$. Then it holds $T A(v) \subset T A(\mu)$, also $T E(v) \subset T E(\mu)$.

We say $\mu \in M(G)$ is symmetric if it holds $\mu(A)=\mu\left(A^{-1}\right)$ for every $A \in \mathscr{B}(G)$.

Proposition 10. If $\mu$ is symmetric, we have $R A(\mu)^{-1}=L A(\mu)$ and $R E(\mu)^{-1}$ $=L E(\mu)$.

Proof. Let $x \in R A(\mu)$, then $\mu(A)=0$ implies $\mu\left(A x^{-1}\right)=0$. By the symmetricity of $\mu, \mu\left(A^{-1}\right)=0$ implies $\mu\left(x A^{-1}\right)=0$. Since $\mathscr{B}(G)=\left\{A^{-1} ; A \in \mathscr{B}(G)\right\}$, we have $L_{x^{-1}}(\mu) \ll \mu$. This shows $x^{-1} \in L A(\mu)$.

Corollary 3. Let $G$ be abelian and $\mu \in M(G)$ be symmetric, then $T A(\mu)$ is a group.

\section{Quasi-invariant measure}

First we prove the Borel measurability of $R A(\mu), R E(\mu), \ldots$ etc. In this section we assume $G$ is separable (= second countable).

Lemma 3. Let $\mu \in M(G)$ and $U$ be an open subset of $G$. Then the functions

(1) $x \longrightarrow \mu\left(U x^{-1}\right)$, and (2) $x \longrightarrow \mu\left(x^{-1} U\right)$

are lower semi-continuous.

Proof. Since $G$ is separable $\mu$ is a $\tau$-smooth measure (see Amemiya, Okada and Okazaki [1]). Hence by the way similar to Amemiya, Okada and Okazaki [1], Lemma 9.4 and Bourbaki [2], § 2, $n^{\circ} 6$, Proposition 11 the assertion follows. In fact remark the following equality;

$$
\mu\left(x^{-1} U\right)=\int_{G} \chi_{\psi^{-1}(U)}(x, y) d \mu(y),
$$

where $\psi(x, y)=x y$ and $\chi_{A}$ is the characteristic function of the Borel set $A$.

This proves the lemma.

Following Dubins and Freedman [4], we put the following Borel structure $\Sigma$ on $M(G)$. The $\sigma$-algebra $\Sigma$ on $M(G)$ is the one generated by the functions

$$
M(G) \ni \mu \longrightarrow \mu(A) \in[0,1]
$$

where $A$ varies over all Borel subsets of $G$. Since $G$ is separable there is a counta- 
ble generator $\left\{A_{n}\right\}$ of $\mathscr{B}(G)$. We may assume $\left\{A_{n}\right\}_{n=1}^{\infty}$ is an algebra. So $\Sigma$ is generated by the countable functions $\mu \rightarrow \mu\left(A_{n}\right) n=1,2, \ldots$.

Proposition 11. Let $\Sigma_{0}$ be the $\sigma$-algebra on $M(G)$ generated by the functions

$$
M(G) \ni \mu \longrightarrow \int_{G} f d \mu, \quad f \in C^{b}(G),
$$

where $C^{b}(G)$ be the space of all bounded continuous functions on $G$. Then it holds $\Sigma_{0}=\Sigma$.

Proof. First we prove $\Sigma \subset \Sigma_{0}$. It is sufficient to show the function $\mu \rightarrow$ $\mu(U)$ is $\Sigma_{0}$-measurable for every open subset $U$ since $G$ is separable. We can take a sequence of closed subsets $F_{n}$ such that $F_{n} \uparrow U$ by the metrizability of $G$. For each $F_{n}$, let $f_{n} \in C^{b}(G)$ be such that $f_{n}=1$ on $F_{n}, f_{n}=0$ on $U^{c}$ and $f_{n} \geqq 0$. Then for every $\mu$ in $M(G)$ we have

$$
\mu(U)=\lim _{n} \int_{G} f_{n} d \mu,
$$

which shows $\mu \rightarrow \mu(U)$ is $\Sigma_{0}$-measurable.

Conversely let $f$ be any bounded continuous function on $G$. Then by Bourbaki [2], $\S 2, n^{\circ} 6$, Lemma 3 , there is a sequence $f_{n}$ of step functions which are the linear combinations of characteristic functions of open subsets such that $f_{n} \uparrow f$ in $G$. For every $\mu$ in $M(G)$, we have

$$
\int_{G} f d \mu=\lim _{n} \int f_{n} d \mu
$$

Since $\mu \rightarrow \int f_{n} d \mu$ is $\Sigma$-measurable, $\mu \rightarrow \int f d \mu$ is also $\Sigma$-measurable, which shows $\Sigma_{0} \subset \Sigma$.

This completes the proof.

Remark 1. $M(G)$ is a separable metric space with respect to the weak *-topology $\sigma\left(M(G), C^{b}(G)\right)$ since $G$ is separable. So the $\sigma$-field $\Sigma$ coincides with the Borel field for the weak *-topology

Lemma 4. For every Borel set $A \in \mathscr{B}(G)$ the functions

$$
x \longrightarrow \mu\left(A x^{-1}\right), \quad x \longrightarrow \mu\left(x^{-1} A\right)
$$

are $\mathscr{B}(G)$-measurable. 
Proof. Let $\mathscr{A}$ be the algebra generated by an open base. Then for every $B$ in $\mathscr{A}$, the functions

$$
x \longrightarrow \mu\left(B x^{-1}\right), \quad x \longrightarrow \mu\left(x^{-1} B\right)
$$

are $\mathscr{B}(G)$-measurable by lemma 3 Since the family $\mathscr{A}$ generates $\mathscr{B}(G)$, the assertion follows.

Proposition 12. The following subsets are Borel sets; $R A(\mu), L A(\mu)$, $T A(\mu), R E(\mu), L E(\mu), T E(\mu), R S(\mu), L S(\mu)$ and $T S(\mu)$, where $\mu \in M(G)$.

Proof. We show $R A(\mu)$ is a Borel set. Let $\psi_{\mu}: G \rightarrow M(G) \times M(G)$ be defined by

$$
\psi_{\mu}(x)=\left(R_{x}(\mu), \mu\right) .
$$

Then $\psi$ is $\mathscr{B}-\Sigma \otimes \Sigma$-measurable, since

$$
x \longrightarrow\left(R_{x}(\mu)(A), \mu(B)\right)=\left(\mu\left(A x^{-1}\right), \mu(B)\right)
$$

is measurable for every $A, B \in \mathscr{B}(G)$. By Dubins and Freedman [4], 2.11, the set $D=\{(\lambda, v) ; \lambda \ll v\}$ is in $\Sigma \otimes \Sigma$, hence $\psi^{-1}(D)=R A(\mu)$ is in $\mathscr{B}(G)$.

The other cases are analogously proved by Dubins and Freedman [4], 2.1.

Let $E$ be a subset of $G$. A measure $\mu \in M(G)$ is said to be right $E$-quasiinvariant (resp. left $E$-quasi-invariant) if it holds $E \subset R A(\mu)$ (resp. $E \subset L A(\mu)$ ). In case $E=G$, we say $\mu$ is right quasi-invariant (resp. left quasi-invariant).

Proposition 13. Let $\mu_{1} \in M(G)$ be right quasi-invariant and $\mu_{1}\left(L A\left(\mu_{2}\right)^{-1}\right)$ $>0$, then it holds $\mu_{2} \ll \mu_{1}$.

Proof. We adopt a way similar to Umemura [8], Proposition 4.1. For every $A \in \mathscr{B}(G)$ we have

$$
\begin{aligned}
\mu_{1} * \mu_{2}(A) & =\int \chi_{A}(x y) d \mu_{1}(x) d \mu_{2}(y) \\
& =\int \mu_{1}\left(A y^{-1}\right) d \mu_{2}(y) \\
& =\int \mu_{2}\left(x^{-1} A\right) d \mu_{1}(x) .
\end{aligned}
$$

Thus $\mu_{1}(A)=0$ implies $\mu_{1} * \mu_{2}(A)=0$, so $\mu_{2}\left(x_{0}^{-1} A\right)=0$ for some $x_{0} \in L A\left(\ddot{\mu}_{2}\right)^{-1}$. 
Since $x_{0}^{-1} \in L A\left(\mu_{2}\right)$, it holds $\mu_{2}(A)=\mu_{2}\left(x_{0} x_{0}^{-1} A\right)=L_{x_{0}^{-1}}\left(\mu_{2}\right)\left(x_{0}^{-1} A\right)=0$, which shows $\mu_{2} \ll \mu_{1}$.

Corollary 4. Let $\mu_{1} \in M(G)$ be right quasi-invariant and $\mu_{2} \in M(G)$ be left quasi-invariant. Then we have $\mu_{1} \sim \mu_{2}$.

Corollary 5. Suppose $G$ is abelian and $\mu \in M(G)$ is right quasi-invariant. Then for every $a \in G$, it holds $R_{a}(\mu) \sim \mu$, that is $R E(\mu)=G$.

Proof. We remark $R_{a}(\mu)$ is also right quasi-invariant for every $a \in G$.

Corollary 6 . Let $G$ be a locally compact topological group and $\mu \in M(G)$ be right (or left) quasi-invariant. Then $\mu$ is equivalent to the Haar measure of $G$. The above is a result of Brockett [3], Theorem 5.

Proposition 14. Let $\mu \in M(G)$ be a right quasi-invariant measure. Then there is a $\mu_{0} \in M(G)$ such that $\mu \sim \mu_{0}$ and $\mu_{0}$ is invariant under the translation of $G$, that is $R_{a}\left(\mu_{0}\right)=\mu_{0}$ for every $a \in G$.

Proof. Since $G$ is separable, the Borel field $\mathscr{B}(G)$ is countably generated and it holds $\mathscr{B}(G) \otimes \mathscr{B}(G)=\mathscr{B}(G \times G)$. Since $d R_{a}(\mu) / d \mu(x)$ can be taken $\mu \otimes \mu$ measurable (Mackey [6], Lemma 3.1), the result follows by the way similar to Umemura [8], Proposition 6.2, Mackey [7], Lemma 7.3.

\section{Size of the set of admissible translates}

Theorem 1. Let $G$ be a separable topological group. Then the subsets $R E(\mu), L E(\mu)$ and $T E(\mu)$ are Borel subgroups. Moreover if $\mu$ is a Radon measure then it hold

(1) $\mu(R E(\mu))=0$, or $R E(\mu)$ is a locally compact group and the restriction $\mu \mid R E(\mu)$ is equivalent to the Haar measure on $R E(\mu)$,

(2) $\mu(L E(\mu))=0$, or $L E(\mu)$ is a locally compact group and $\mu \mid L E(\mu)$ is equivalent to the Haar measure on $L E(\mu)$.

Proof. (1) Assume $\mu(R E(\mu))>0$. Then $v=\mu \mid R E(\mu)$ is a right quasiinvariant measure on $R E(\mu)$. Thus there is a right invariant Radon measure $\lambda$ on $R E(\mu)$ so that $v \sim \lambda$ (Proposition 14). By Gowrisankaran [5], Corollary 1, or Weil [9], Appendix $1, R E(\mu)$ is locally compact group and $\lambda$ is equivalent to the Haar measure (Corollary 6). (2) is analogous to (1).

This proves the theorem. 
Theorem 2. Let $G$ be a separable abelian topological group and $\mu$ be a symmetric Radon probability measure on $G$. Then $\mu(T A(\mu))=0$, or $T A(\mu)$ is a locally compact group and $\mu \mid T A(\mu)$ is equivalent to the Haar measure on $T A(\mu)$.

Proof. By corollary 3, $T A(\mu)$ is a Borel subgroup. By the way same to theorem 1 , the theorem is proved.

REMARK 2. Let $G$ be a complete separable metric group. In theorem 2, if $\mu$ is not symmetric we consider the symmetrization $v, v=\mu * \mu^{2}$, where $\mu^{2}(A)$ $=\mu\left(A^{-1}\right)$. Then by proposition 9 we have $T A(\mu) \subset T A(v)$ and $T A(v)$ is a Borel subgroup (Corollary 3 and proposition 12). Thus if $\mu(T A(\mu))>0$, we have

$$
\begin{aligned}
v(T A(v)) & \geqq v\left(T A(\mu) T A(\mu)^{-1}\right) \geqq \int_{T A(\mu)} \mu\left(T A(\mu) T A(\mu)^{-1} x\right) d \mu(x) \\
& \geqq \mu(T A(\mu)) \mu(T A(\mu))>0 .
\end{aligned}
$$

By theorem 2, $T A(v)$ is a locally compact group and $T A(\mu) \subset T A(v)$.

REMARK 3. In theorems 1 and 3, if $\mu$ is only a Borel measure we can show $R E(\mu), T A(\mu)$ is a dense subgroup of a locally compact group. It is sufficient to consider the completion $\hat{G}$ of $G$ and use proposition 7 .

\section{References}

[1] I. AmemiYA, S. OKada and Y. OKazAKI, Pre-Radon measures on topological spaces, Kodai Math. J., Vol. 1 (1978), 101-132.

[2] N. Bourbaki, Intégration, Chapitre 9, Hermann, Paris (1969).

[3] P. L. BRockeTt, Admissible transformations of measures, Semigroup Forum, Vo.1 12 (1976), 21-33.

[4] L. Dubins and D. Freedman, Measurable sets of measures, Pacific J. Math., 14 (1964), 1211-1222.

[5] C. Gowrisankaran, Radon measures on groups, Proc. A.M.S., 25 (1970), 381-384.

[6] G. W. MAckeY, A theorem of Stone and von Neumann, Duke Math. J., 16 (1949), 313-326.

[7] G. W. MACKeY, Borel structure in groups and their duals, Trans. Amer. math. Soc., 85 (1957), 134-165.

[8] Y. Umemura, Measures on infinite dimensional vector spaces, Publ. R.I.M.S., Ser. A, 1 (1965), 1-47.

[9] A. WeIL, L'integration dans les groupes topologiques et ses applications, Hermann, Paris (1951).

[10] J. ZINN, Admissible translates of stable measures, Studia Math., T. LIV (1976), 245-257. 